\title{
The ZZ/ZW sex-determining mechanism originated twice and independently during evolution of the frog, Rana rugosa
}

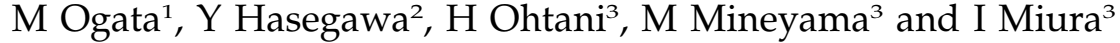 \\ ${ }^{1}$ Laboratory of Zoo Biology, Preservation and Research Center, Yokohama, Japan; ${ }^{2}$ Institute for Comprehensive Medical Science, \\ Fujita Health University, Toyoake, Japan and Institute for Amphibian Biology, Graduate School of Science, Hiroshima University, \\ Higashi-Hiroshima, Japan
}

\begin{abstract}
The Japanese frog, Rana rugosa, has two distinct sex chromosome types, $\mathrm{XX} / \mathrm{XY}$ and ZZ/ZW. These two types are found in localized groups, separated geographically by a boundary area predicted to lie somewhere around Lake Biwa in central Japan. To determine this precise boundary, the heterogametic sex of 18 populations around Lake Biwa was examined by genotyping sex-linked genes. Phylogenetic relationships between the populations were also analyzed using mitochondrial 12S rRNA gene. Results showed that the Suzuka-Kii mountain range located east of Lake Biwa separated the $X X / X Y$ populations from the $Z Z / Z W$ populations. Unexpectedly, from a phylogenetic perspective, the
\end{abstract}

ZZ/ZW populations around Lake Biwa belonged not to the main $Z W$ group but to the $X Y$ group. The authors propose that the ZZ/ZW populations around Lake Biwa diverged secondarily from the $X X / X Y$ group through a change of heterogametic sex, eventually forming a new group. This group was thus named the 'Neo-ZW group'. As the main ZW group inhabiting northwestern Japan is known to have a different male heterogametic origin, this finding shows that change of heterogametic sex from male to female may have occurred twice, and independently, during the frog speciation.

Heredity (2008) 100, 92-99; doi:10.1038/sj.hdy.6801068; published online 14 November 2007

Keywords: frog; sex chromosomes; heterogametic sex; sex determination; Rana

\section{Introduction}

Genetic sex determination in animals and plants follows two main types of heterogamety: male heterogamety $(X X / X Y)$ and female heterogamety $(\mathrm{ZZ} / \mathrm{ZW})$. In vertebrates, the two types are shared by the taxonomic groups and, particularly in lower vertebrates, they are shared even by closely related species (Bull, 1983; Ezaz et al., 2006). It has been shown in amphibians that the heterogametic sex was originally female, and subsequently changed more than once to male at certain branching points in amphibian phylogenetic evolution (Hillis and Green, 1990). In addition, there is evidence that the heterogametic sex returned to its previous female state in the frog Rana rugosa when its distribution range expanded across Japan (Miura et al., 1998; Ogata et al., 2002, 2003). Therefore, it is plausible that heterogametic sex is not permanently fixed, but can change during evolution and speciation. To understand the evolutionary mechanisms of sex determination, it is important to determine the evolutionary reasons for and mechanisms of heterogametic sex change.

Correspondence: Dr M Ogata, Laboratory of Zoo Biology, Preservation and Research Center, Yokohama, 155-1 Kawaijuku-cho, Asahi-ku, Yokohama, Japan.

E-mail:ZVP06246@nifty.ne.jp

Received 3 April 2007; revised 16 September 2007; accepted 19

September 2007; published online 14 November 2007
Rana rugosa is found in four countries bordering the Sea of Japan: China, North and South Korea, and Japan. Japanese Rana populations are divided into four genetic groups that inhabit four different geographic regions (Figure 1). These groups differ in both sex determination and sex chromosome differentiation. The ZW group, distributed in north-west Japan, has female heterogametic sex determination with differentiated sex chromosomes. In contrast, the west Japan, Kanto (around Tokyo) and $X Y$ groups have male heterogametic sex determination, the former two of which have homomorphic sex chromosomes in both sexes while the latter XY group has differentiated sex chromosomes (Nishioka et al., 1994). The $X / Y$ and Z/W differentiated sex chromosomes are all seventh largest in the chromosome complement $(2 n=26)$. On the basis of previous studies, it is hypothesized that the two sex-chromosome-differentiated $X Y$ and $Z W$ groups share their origins at hybridization between the primary populations of the west Japan and Kanto groups (that have undifferentiated sex chromosomes) (Ogata et al., 2003). The main reason for this hypothesis is that the sex chromosomes are chimeric; Z and Y chromosomes are almost morphologically identical in terms of the shape of mitotic and meiotic lampbrush chromosomes and in their banding pattern. Their sex-linked ADP/ATP translocase $(A A T)$ sequence is similar to the sequence for chromosome 7 of the west Japan group, suggesting a shared origin in chromosome 7 of the west Japan group. In contrast, W 


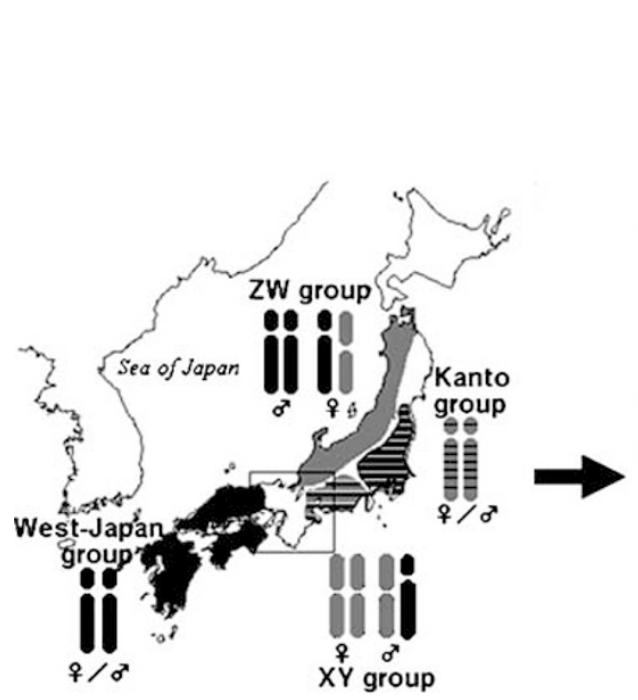

Figure 1 The left-hand map shows four geographic groups of Rana rugosa in Japan, and the right-hand map shows the localities of the 18 populations examined. The southern ends of the distribution of the ZW (gray zone) and XY (gray zone with horizontal lines) groups come into contact with each other around Lake Biwa (squared area on the left-hand figure). The circles on the map denote the southernmost populations of the ZW and XY groups examined to date. The right-hand figure is a magnification of the central area of Japan. Abbreviations are listed in Table 1. The Katata population, indicated by an open circle, is exceptional as it is heterogametic in females, although it belongs to the $\mathrm{XY}$ group phylogenetically.

and $\mathrm{X}$ chromosomes have their origins in chromosome 7 of the Kanto group (Miura et al., 1996, 1997, 1998; Ohtani et al., 2000). Artificial crossing between the parental west Japan and Kanto groups showed sex ratio skewing in the offspring. Thus, sex ratio distortion caused by hybridization is assumed to have been the trigger that induced sex chromosome differentiation (Ogata et al., 2003).

To prove the above hypothesis and understand the mechanisms of heterogametic sex change, it is necessary to approach and identify boundary regions between the geographic groups and examine the phylogenetic history. In addition, it is very interesting to examine the mutual affinity of the two different systems of sex determination when they meet secondarily after diverged from each other. To date, there is just one set of data suggesting the geographic location of the boundary area between the $X Y$ and ZW groups. The Katata population located near the center of Japan on the west side of Lake Biwa in Shiga prefecture, has ZZ/ZW sex chromosomes but belongs genetically to the XY group (Nishioka et al., 1993a, 1994). The existence of this population suggests previous mingling of the $\mathrm{XY}$ and $\mathrm{ZW}$ groups. Therefore, in the present study, we focused on the 18 populations in the area around Lake Biwa, and determined their heterogametic sex by genotyping sex-linked genes to identify the precise boundary area between the $\mathrm{XY}$ and $\mathrm{ZW}$ groups. Genotyping of mitochondrial $12 S$ rRNA gene was carried out to examine their phylogenetic relationships. As a result, we identified the geographic boundary region, located east of Lake Biwa, which separates populations with $\mathrm{ZZ} / \mathrm{ZW}$ sex determination from those with $X X / X Y$ sex determination. Several populations in which two different sex-determining systems are mingling were also discovered. Unexpectedly, the populations with $\mathrm{ZZ} / \mathrm{ZW}$ sex determination were found to belong genetically to the $X Y$ groups not to the main $Z W$ group. They thus constitute a new geographic group, suggesting their very recent deviation from the $\mathrm{XY}$ group through change of heterogametic sex. A plausible evolutionary history to explain the establishment of this new group is presented.

\section{Materials and methods}

\section{Frogs}

The 165 frogs in total were collected from 18 points around Lake Biwa located in almost the center of Japan, at the breeding season (June) from 1994 to 2006 (Figure 1; Table 1). Sex of all frogs was examined according to the external morphology of the gonads.

\section{Genotyping analyses of sex-linked genes}

Total RNA was extracted from the forelimb fingers of each frog using SV total RNA isolation system (Promega, WI, Madison, USA) according to the manufacturer's instruction. First strand cDNA was synthesized from $1 \mu \mathrm{g}$ of the total RNA using $\mathrm{dT}_{24}$ oligomer and reversetranscriptase (SuperScript II, BRL, New York, USA), basically according to the method described in Ogata et al. (2003). For restriction fragment-length polymorphism analyses of sex-linked genes, AAT (ADP/ATP translocase, Miura et al., 1998) and $A R$ (androgen receptor, Ohtani et al., 2003), they were amplified from the $1 \mu \mathrm{l}$ of cDNA at 37 cycles of $94^{\circ} \mathrm{C} 40 \mathrm{~s}, 62^{\circ} \mathrm{C} 40 \mathrm{~s}$, and $72{ }^{\circ} \mathrm{C} 30 \mathrm{~s}$, ending with $72{ }^{\circ} \mathrm{C} 2 \mathrm{~min}$ in a $50 \mu \mathrm{l}$ reaction solution containing $5 \mu \mathrm{l}$ of $10 \times$ Ex buffer, $4 \mu \mathrm{l}$ of $2.5 \mathrm{mM}$ dNTP, $0.3 \mu \mathrm{l}$ of Ex Taq (Takara, Tokyo, Japan) and the following primers: for $A A T$, forward $5^{\prime}$-CCTTGTG CTTCGTTTACCCAC-3' and reverse $5^{\prime}$-CCTGCTACCCT CATCTCTTGCA-3' ${ }^{\prime}$, and for AR, forward $5^{\prime}$-GCGGAAA TGTTATGAGGCTGG-3' and reverse $5^{\prime}$-AAAATCCAC GCTCACCATCTG-3'.

Next, $15 \mu \mathrm{l}$ of the amplified product was cut with $\mathrm{MboI}$ for $2 \mathrm{~h}$ at $37^{\circ} \mathrm{C}$ for $A A T$, and with TaqI for $1 \mathrm{~h}$ at $65^{\circ} \mathrm{C}$ for $A R$, respectively, and electrophoresed in agarose gel (3\%) with TAE buffer ( $40 \mathrm{mM}$ Tris-acetate, $1 \mathrm{mM}$ EDTA). To amplify the genomic $A A T$, we used $500 \mathrm{ng}$ genomic DNA as the template and the following primers: the same $A A T$ forward primer and the reverse $5^{\prime}$-AGTTCACCC ACCTTTAGCTG- $3^{\prime}$, harboring $3^{\prime}$ end of the one exon and $5^{\prime}$-end of the next intron (Sakisaka et al., 2000). Since the 543rd nucleotide of $A A T$ of $\mathrm{W}$ and $\mathrm{X}$ chromosomes 
Table 1 Populations and specimens

\begin{tabular}{|c|c|c|c|c|c|}
\hline \multirow[t]{2}{*}{ Population } & \multicolumn{2}{|c|}{ No. of frogs } & \multirow[t]{2}{*}{ Prefecture } & \multirow[t]{2}{*}{ Station } & \multirow[t]{2}{*}{ Collection year } \\
\hline & ơ & q & & & \\
\hline P1: Gifu & 2 & 1 & Gifu & Yamagata-city, Ijira-cho & 2005,2006 \\
\hline P2: Sekigahara & 3 & 5 & Gifu & Fuwa-gun, Sekigahara-cho & 2004 \\
\hline P3: Kameyama & 1 & 9 & Mie & Kameyama-city, Shirakiishiki-cho & 2006 \\
\hline P4: Kasagi & 6 & 5 & Mie & Ohki-cho, Saki & 2006 \\
\hline P5: Mikata & 5 & 1 & Fukui & Mikatakaminaka-gun, Wakasa-cho & 1994 \\
\hline P6: Kinomoto & 5 & 5 & Shiga & Ika-gun, Kinomoto-cho & 2004 \\
\hline P7: Eigenji & 2 & 8 & Shiga & Higashiohmi-city, Eigenjitakano-cho & 2004 \\
\hline P8: Watsuka & 7 & 5 & Kyoto & Sagara-gun, Watsuka-cho & 2004,2005 \\
\hline P9: Kounoyama & 11 & 2 & Nara & Yamazoe-village & 2006 \\
\hline P10: Haibara & 8 & 7 & Nara & Uda-city, Haibara & 2006 \\
\hline P11: Shimokitayama & 4 & 3 & Nara & Shimokitayama-village & 2006 \\
\hline P12: Takashima & 2 & 9 & Shiga & Takashima-city, Noda & 2004 \\
\hline P13: Kyoto & 3 & 3 & Kyoto & Kyoto-city, Higashiyama-ward & 2004 \\
\hline P14: Ikoma & 9 & 4 & Nara & Ikoma-gun, Heguri-cho & 2006 \\
\hline P15: Kyoshi & 3 & 8 & Osaka & Sennan-gun, Misaki-cho & 2006 \\
\hline P16: Kawabe & 3 & 8 & Wakayama & Hidaka-gun, Hidakagawa-cho & 2006 \\
\hline P17: Awaji & 3 & 3 & Hyogo & Awaji-city, Sumoto & 2006 \\
\hline P18: Okayama & 1 & 1 & Okayama & Tamano-city & 2006 \\
\hline
\end{tabular}

gives $M b o$ I site whereas the corresponding site (550th) of $\mathrm{Z}$ and $\mathrm{Y}$ chromosomes does not, $\mathrm{MboI}$ digestion of the amplified product allows discrimination of $W / X$ alleles from Z/Y alleles (Sakisaka et al., 2000). As for AR, we amplified the AR fragment using the above primers from $\mathrm{ZZ}$ and WW cDNA, sequenced and identified one nucleotide difference between them and found that it gives TaqI site to $\mathrm{Z} / \mathrm{Y}$ alleles but not to $\mathrm{W} / \mathrm{X}$ alleles. The sex linkage is confirmed using genomic southern hybridization (unpublished) and is partly proved by Ohtani et al. (2003).

Degenerate oligonucleotide-primed PCR of WW and ZZ DNA by Miura et al. (unpublished) allowed WWspecific amplification of a transposon-like sequence. To amplify alleles specific to the $W$ and $X$ chromosomes, we designed the following primers and amplified at 30 cycles of $94{ }^{\circ} \mathrm{C} 40 \mathrm{~s}, 60^{\circ} \mathrm{C} 40 \mathrm{~s}$ and $72{ }^{\circ} \mathrm{C} 1 \mathrm{~min}$, ending with $72{ }^{\circ} \mathrm{C} 2 \mathrm{~min}$ : forward $5^{\prime}$-ATGTGGCCATTCT CCCTTCCTG-3' and reverse 5'-ATGTGGTGTGGCTG TTTCAAGC-3'. Using the primers only $W$ and $X$ chromosome alleles can be amplified.

\section{Mitochondiral DNA}

Total genomic DNA was isolated from blood cells using DNA WB extractor (Wako, Japan) according to the manufacturer's instruction. Mitochondrial 12S rRNA gene was amplified from $500 \mathrm{ng}$ of the total genomic DNA at 35 cycles of $94{ }^{\circ} \mathrm{C} 40 \mathrm{~s}, 58^{\circ} \mathrm{C} 1 \mathrm{~min}, 72{ }^{\circ} \mathrm{C} 1 \mathrm{~min}$, ending with $72{ }^{\circ} \mathrm{C} 2 \mathrm{~min}$ in a $50 \mu \mathrm{l}$ reaction solution containing Ex Taq (Takara) and the following primers: forward 5'-CAAGTCTCAGCACACCCGT-3' and reverse 5'-TGTTACGACTTGCCTCTTCTA-3'. The amplified products were cut with $\operatorname{HinfI}$ at $37{ }^{\circ} \mathrm{C}$ for $2 \mathrm{~h}$ and were electrophoresed in agarose gel $(3 \%)$ with TAE buffer. To discriminate haplotype of XY group from those of ZW and west Japan groups, we utilized 206th nucleotide difference giving HinfI site to the XY haplotype but not to those of the other two groups (Ogata et al., 2003). In addition, we determined full nucleotide sequence of the amplified products from one male and one female of each population and confirmed sequence agreement between the same haplotypes and disagreement between the different haplotypes.

\section{Artificial crossing and diploid gynogenesis}

Artificial crossing was performed between the frogs of Hiroshima population of the west Japan group and those of Hamakita population of the XY group according to the method of Ohtani et al. (1997). Gynogenetic diploid embryos were produced from two females of Kyoto population using the method of Ohtani et al. (1997). Sex chromosome constitution of the gynogenetic diploids was checked on the basis of the $A A T$ genotypes.

\section{Results}

\section{Genotyping of AAT and AR sex-linked genes}

The W, X, Y and Z sex chromosomes in Rana rugosa are all the seventh largest in the complement $(2 n=26)$. The $W$ chromosome has an origin common with $X$, and $Z$ has an origin in common with Y. These common origins are reflected in the similarity of both morphology and the sex-linked gene sequences between $W$ and $X$, and $Z$ and Y (Miura et al., 1998). Restriction fragment-length polymorphism analyses of $A A T$ and $A R$ discriminate between the $\mathrm{W}$ and $\mathrm{Z}$ chromosomes and between the $\mathrm{X}$ and $Y$ chromosomes (Miura et al., 1998; Sakisaka et al., 2000; Ohtani et al., 2003). In the ZZ/ZW type, ZZ male and $Z W$ female, respectively, are shown by homozygous bands $a / a$ and heterozygous bands $a / b$, while in the XX/ $X Y$ type, $X X$ female and $X Y$ male, respectively, are shown by bands $b / b$ and $b / a$ (Figure 2). The results of $A A T$ and $A R$ genotyping for the 18 populations around Lake Biwa are shown in Tables 2 and 3 . It is evident that four populations (P1-P4) are heterogametic in males while seven populations (P5, P6 and P11-P15) are heterogametic in females. However, in the remaining four populations (P7-P10) two kinds of heterogametic sex may be mingling (Table 2). In the P7 (Eigenji) population, one of two males was heterozygous $(a / b)$ for $A A T$ and 
one of eight females was homozygous $(b / b)$, suggesting that they are $X Y$ male and $X X$ female, respectively. The same situation was observed in the P9 and P10 populations. Likewise, in the P8 (Watsuka) population, three out of seven males were homozygous $(a / a)$ for $A A T$, suggesting that the males are homogametic $\mathrm{ZZ}$ males (Figure 3). However, since the genotypes of $A A T$ and $A R$ do not coincide with each other in the three males (nos. 2, 3 and 7), we further examined the genotypes of genomic $A A T$ as well as the $\mathrm{W} / \mathrm{X}$-specific transposon sequence that is successfully amplified from the $\mathrm{W}$ and $\mathrm{X}$ chromosomes but not from the $\mathrm{Z}$ or $\mathrm{Y}$ chromosomes to confirm the sex chromosome constitution of the three males (Table 4). The AAT genotype of genomic DNA was identical to that of cDNA. The $\mathrm{W} / \mathrm{X}-$ specific transposon coincided with allele $b$ of $A A T$ but not with allele $b$ of $A R$ in two of the males (nos. 2 and 3). We assume that the $A A T$ and $\mathrm{W} / \mathrm{X}$ transposon loci are closely linked with a sex-determining locus, but that

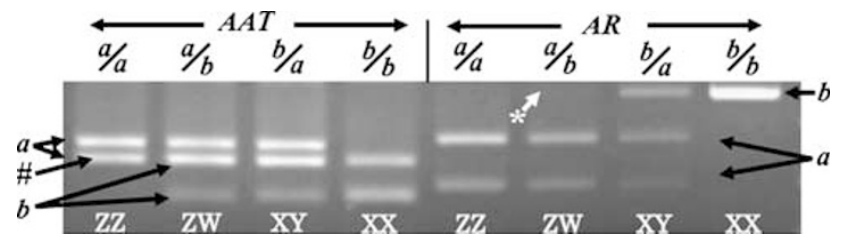

Figure $2 A A T$ and $A R$ genotypes in the $Z \mathrm{~W}$ and $\mathrm{XY}$ groups. $\mathrm{ZZ}$ male and $\mathrm{ZW}$ female are shown by the $a / a$ and $a / b$ bands, respectively, while the XY male and XX female are shown by the $b / a$ and $b / b$ bands, respectively. *indicates allele $b$ whose expression is extremely low and thus the band is not visible, giving an apparent homozygous $a / a$ genotype. "indicates an $A A T$ band appearing in both sexes, which appears because alleles $a$ and $b$ both have an MboI site, at the 656th nucleotide of $A A T$ on the $\mathrm{W}$ and $\mathrm{X}$ chromosomes, and at the 663rd nucleotide of $A A T$ on the $\mathrm{Z}$ and $\mathrm{Y}$ chromosomes (Miura et al., 1998). their linkage with $A R$ may have been broken through some structural change in the population. However, we do not yet have sufficient data to speculate the reason why this might occur. Thus, males 1,2 and 3 might be ZZ males (not XY males) based on the genotypes of $A A T$ and $W / X$-specific transposon while male 7 may be an $X Y$

Table 3 Genotypes of $A R$

\begin{tabular}{|c|c|c|c|c|c|c|c|c|}
\hline \multirow[t]{3}{*}{ Population } & \multicolumn{6}{|c|}{ AR } & \multirow[t]{3}{*}{ Total } & \multirow{3}{*}{$\begin{array}{l}\text { Heterogametic } \\
\text { sex }\end{array}$} \\
\hline & \multicolumn{3}{|c|}{$\widehat{0}$} & \multicolumn{3}{|c|}{ q } & & \\
\hline & \multicolumn{6}{|c|}{$\mathrm{a} / \mathrm{a} a / b \mathrm{~b} / \mathrm{b} a / \mathrm{a} a / b \mathrm{~b} / \mathrm{b}$} & & \\
\hline P1: Gifu & 0 & 2 & 0 & 0 & 0 & 1 & 3 & Male \\
\hline P2: Sekigahara & 0 & 3 & 0 & 0 & 0 & 5 & 8 & Male \\
\hline P3: Kameyama & 0 & 1 & 0 & 0 & 0 & 9 & 10 & Male \\
\hline P4: Kasagi & 0 & 6 & 0 & 0 & 0 & 5 & 11 & Male \\
\hline P5: Mikata & 5 & 0 & 0 & $1^{\mathrm{a}}$ & 0 & 0 & 6 & Female \\
\hline P6: Kinomoto & 5 & 0 & 0 & 0 & 5 & 0 & 10 & Female \\
\hline P7: Eigenji & 1 & 1 & 0 & 0 & 7 & 1 & 10 & Female/male \\
\hline P8: Watsuka & 1 & $\underline{5}$ & 1 & 0 & 0 & 5 & 12 & Male/female \\
\hline P9: Kounoyama & $\overline{8}$ & $\overline{3}$ & $\overline{0}$ & 0 & 1 & 1 & 13 & Female/male \\
\hline P10: Haibara & 7 & 1 & 0 & 0 & 6 & 1 & 15 & Female/male \\
\hline P11: Shimokitayama & 4 & 0 & 0 & 0 & 2 & $\underline{1}$ & 7 & Female \\
\hline P12: Takashima & 2 & 0 & 0 & 0 & 9 & $\overline{0}$ & 11 & Female \\
\hline P13: Kyoto & 3 & 0 & 0 & 0 & 3 & 0 & 6 & Female \\
\hline P14: Ikoma & 8 & 1 & 0 & 0 & 4 & 0 & 13 & Female \\
\hline P15: Kyoshi & 3 & $\overline{0}$ & 0 & 0 & 8 & 0 & 11 & Female \\
\hline P16: Kawabe & 3 & 0 & 0 & 8 & 0 & 0 & 11 & $?$ \\
\hline P17: Awaji & 2 & 1 & 0 & 3 & 0 & 0 & 6 & Male $^{\mathrm{b}}$ \\
\hline P18: Okayama & 1 & $\overline{0}$ & 0 & 1 & 0 & 0 & 2 & Male $^{\mathrm{b}}$ \\
\hline
\end{tabular}

$A R$ genotypes showing heterogametic sex are indicated in bold. $A R$ genotypes showing disagreement with $A A T$ genotypes are underlined.

${ }^{a}$ An apparent homozygosity of $a / a$ in the females because AR expression of the $W$ allele is extremely low (Ohtani et al., 2003).

${ }^{\mathrm{b}}$ Abbreviation is the same as in the Table 2 .

Table 2 AAT genotype and mitochondrial 12S rRNA

\begin{tabular}{|c|c|c|c|c|c|c|c|c|c|}
\hline \multirow[t]{3}{*}{ Population } & \multicolumn{6}{|c|}{ AAT } & \multirow[t]{3}{*}{ Total } & \multirow[t]{3}{*}{ Heterogametic sex } & \multirow[t]{3}{*}{$12 S$ rRNA type } \\
\hline & \multicolumn{3}{|c|}{ 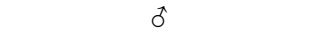 } & \multicolumn{3}{|c|}{ 우 } & & & \\
\hline & $\mathrm{a} / \mathrm{a}$ & $a / b$ & $\mathrm{~b} / \mathrm{b}$ & $\mathrm{a} / \mathrm{a}$ & $a / b$ & $\mathrm{~b} / \mathrm{b}$ & & & \\
\hline P1: Gifu & 0 & 2 & 0 & 0 & 0 & 1 & 3 & Male & $X Y$ \\
\hline P2: Sekigahara & 0 & 3 & 0 & 0 & 0 & 5 & 8 & Male & $X Y$ \\
\hline P3: Kameyama & 0 & 1 & 0 & 0 & 0 & 9 & 10 & Male & $X Y$ \\
\hline P4: Kasagi & 0 & 6 & 0 & 0 & 0 & 5 & 11 & Male & $X Y$ \\
\hline P5: Mikata & 5 & 0 & 0 & 0 & 1 & 0 & 6 & Female & ZW \\
\hline P6: Kinomoto & 5 & 0 & 0 & 0 & 5 & 0 & 10 & Female & $X Y$ \\
\hline P7: Eigenji & 1 & 1 & 0 & 0 & 7 & 1 & 10 & Female/male & $X Y$ \\
\hline P8: Watsuka & 3 & 4 & 0 & 0 & 0 & 5 & 12 & Male/female & $X Y$ \\
\hline P9: Kounoyama & 8 & 3 & 0 & 0 & 1 & 1 & 13 & Female/male & $X Y$ \\
\hline P10: Haibara & 7 & 1 & 0 & 0 & 6 & 1 & 15 & Female/male & $X Y$ \\
\hline P11: Shimokitayama & 4 & 0 & 0 & 0 & 3 & 0 & 7 & Female & $X Y$ \\
\hline P12: Takashima & 2 & 0 & 0 & 0 & 9 & 0 & 11 & Female & $X Y$ \\
\hline P13: Kyoto & 3 & 0 & 0 & 0 & 3 & 0 & 6 & Female & $X Y$ \\
\hline P14: Ikoma & 9 & 0 & 0 & 0 & 4 & 0 & 13 & Female & $X Y$ \\
\hline P15: Kyoshi & 3 & 0 & 0 & 0 & 8 & 0 & 11 & Female & $X Y$ \\
\hline P16: Kawabe & 3 & 0 & 0 & 8 & 0 & 0 & 11 & $?$ & $X Y$ \\
\hline P17: Awaji & 3 & 0 & 0 & 3 & 0 & 0 & 6 & Male $^{\mathrm{a}}$ & West \\
\hline P18: Okayama & 1 & 0 & 0 & 1 & 0 & 0 & 2 & Male $^{\mathrm{a}}$ & West \\
\hline
\end{tabular}

AAT genotypes showing heterogametic sex are indicated in bold.

? mean unknown.

${ }^{a}$ A population presumably bearing male heterogametic sex determination because its $12 \mathrm{~S}$ rRNA sequence is similar to that of the west Japan group, which has male heterogametic sex determination with undifferentiated sex chromosomes. 
male (not XX male). This kind of genotype disagreement between the two sex-linked genes was also observed in the P11, P14 and P17 populations. In these populations, the $\mathrm{W} / \mathrm{X}$ transposon was always linked with allele $b$ of $A A T$, as in the P8 (Watsuka) population (data not shown). In the remaining three populations (P16-P18), the AAT genotypes of all males and females were homozygous (a/a) (Table 2). This is very similar to the AAT genotype of the west Japan group, which is another main geographic group with undifferentiated sex chromosomes (Miura et al., 1998).

\section{Phylogenetic analysis using mitochondrial 12S rRNA gene}

Restriction fragment-length polymorphism analysis of mitochondrial $12 S$ rRNA gene can be used to discriminate between the $Z Z / Z W$ and $X X / X Y$ groups (Ogata et al., 2003). The haplotypes of the 18 populations examined are shown in Table 2. Fifteen populations (P1-P4 and P6-P16) had a XX/XY group haplotype. Four of the populations (P1-4) were heterogametic in males. The P17 and P18 populations showed the same sequence as that of the west Japan group, based on the full sequence determined, and thus belong to the west Japan group. It was not possible to determine the heterogametic sex of the P16 population, as the AAT and $A R$ genotypes were homozygous $a / a$ in both sexes. Surprisingly, six populations (P6 and P11-P15) were heterogametic in females. Thus, their sex determination mechanism does not coincide with their phylogenetic background. In the P7-10 populations, heterogametic males and females were mingling. However, the haplotypes of the P5 population placed them in the ZW
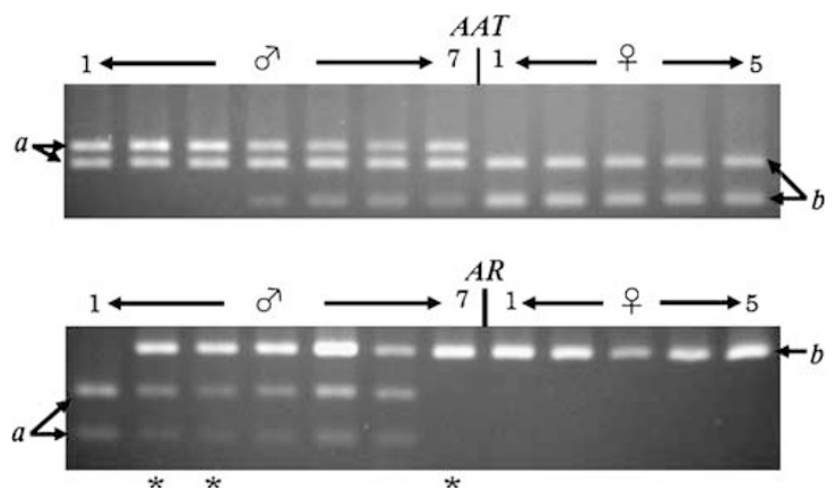

Figure $3 A A T$ and $A R$ genotypes in the P8 Watsuka population *indicates the $A R$ genotype that does not coincide with the $A A T$ genotype. groups, and their heterogametic sex was also female, thus they belong to the main ZW group.

\section{Gynogenetic diploids}

The heterogametic sex of six populations (P6 and P11-15) was female, but the haplotypes of their mitochondrial $12 S$ rRNA were the same as the $X Y$ group. This shows disagreement between sex determination type and genetic background. In addition, the $\mathrm{W}$-borne allele of $A R$ in the main $\mathrm{ZW}$ group is known to have extremely low expression (Ohtani et al., 2003), although it was found that the expression of the $\mathrm{W}$-borne allele in the six populations was as high as that of the Z-borne allele, similar to the $X$-allele of the $X Y$ group. As the $W$ chromosome of the $\mathrm{ZW}$ group is known to have accumulated degenerated genes that cause lethality in WW embryos, we used diploid gynogenesis to create WW embryos from two females of the Kyoto population (P13). Twenty-one viable tadpoles were obtained from female no. 1: 10 bearing the $b / b$ genotype for $A A T$ died within two weeks after the feeding stage, whereas the remaining 11 bearing the $a / a$ genotype completed metamorphosis. Thirteen tadpoles were obtained from female no. 2, all of which completed metamorphosis. Nine of the progeny were $a / a$ for $A A T$ and four were $b / b$. These results indicate that the $\mathrm{W}$ chromosome from the no. 1 female had lethal genes whereas the no. 2 female did not. It is therefore possible to hypothesize that $W$ chromosomal degeneration in the P13 population is at quite an early stage.

\section{Discussion}

\section{Geographic boundary separating the populations with $\mathrm{XX} / \mathrm{XY}$ and $\mathrm{ZZ/ZW}$ sex determination, and a new group 'Neo-ZW'}

Genotyping analyses of the sex-linked $A A T$ and $A R$ genes allowed determination of the heterogametic sex of the 18 populations around Lake Biwa, the region thought to be the boundary area between the $\mathrm{ZZ/ZW}$ and $\mathrm{XX} / \mathrm{XY}$ sex chromosome groups of Rana rugosa. Six populations (P6 and P11-P15) are heterogametic in females, and four (P1$P 4)$ are heterogametic in males. As the $Z Z / Z W$ and $X X /$ $X Y$ sex chromosome groups are found on either side of the Suzuka-Kii mountain range, running north to south, this mountain range was hypothesized as the boundary area separating them (Figure 4). In fact, $X Y$ males and $X X$ females were found together with $\mathrm{ZZ}$ males and $\mathrm{ZW}$ females in four populations (P7-P10) found only at the foot of the western part of the mountain range. This shows that mingling is taking place between two groups

Table 4 Genotypes of $A A T, A R$ and W/X-transposon in the males of Watsuka population (P8)

\begin{tabular}{lccccc}
\hline Male nos. & AAT $(c D N A)$ & AAT (genomic) & AR & W/X transposon & Presumed sex chromosome type \\
\hline 1 & $a / a$ & $a / a$ & $a / a$ & - & $\mathrm{ZZ}$ \\
2 & $a / a$ & $a / a$ & $a / b$ & - & $\mathrm{ZZ}$ \\
3 & $a / a$ & $a / a$ & $a / \bar{b}$ & - & $\mathrm{ZZ}$ \\
4 & $a / b$ & $a / b$ & $a / b$ & + & $\mathrm{XY}$ \\
5 & $a / b$ & $a / b$ & $a / b$ & + & $\mathrm{XY}$ \\
6 & $a / b$ & $a / b$ & $a / b$ & + & $\mathrm{XY}$ \\
7 & $a / b$ & $a / b$ & $\underline{b} / b$ & + & $\mathrm{XY}$ \\
\hline
\end{tabular}

Underlined alleles $b$ of AR are not linked with alleles $b$ of AAT; - indicates 'not amplified' and + 'amplified'. 


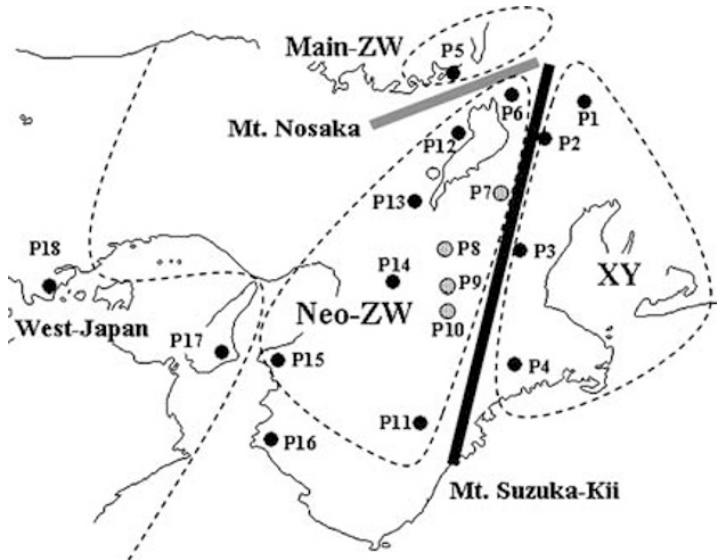

Figure 4 The new group, 'Neo-ZW', is separated from the main $\mathrm{ZW}$ and XY groups by the Nosaka (thick gray line) and Suzuka-Kii (thick black line) mountain ranges, respectively. The dotted circle indicates a population where two kinds of heterogametic sex were found together. The open circle indicates the Katata population.

that have different sex determining mechanisms. The P17 and P18 populations plausibly belong to the west Japan group and their heterogametic sex may be male. The remaining $\mathrm{P} 16$ population is homozygous $a / a$ for $A A T$ and $A R$, both in males and females, as in the P17 and P18 populations, although mitochondrial 12S rRNA sequence shows that, phylogenetically, P16 belongs to the XY group. Since this combination of sex-linked gene haplotype and mitochondrial genotype has never been found in this species, there is no available information regarding what type of sex determination is at work.

Genotyping of $A R$ and mitochondrial $12 S$ rRNA provided information to further divide the seven $\mathrm{ZZ/}$ ZW populations (P5-6 and P11-15) into two different groups. $A R$ expression of the $W$ allele is known to be extremely low in females, far less than that of the $\mathrm{Z}$ allele (Ohtani et al., 2003). In the P5 (Mikata) population, males and females are both homozygous $a / a$ for $A R$. Since the female heterogamety has been proved by $A A T$ genotyping (Table 2), it is evident that the $A R$ expression from the W-borne allele in the P5 population is faint, or indeed absent, as seen in the main ZW group. In contrast, the W-borne allele of $A R$ in the remaining six populations (P6 and P11-P15) is normally expressed like the $Z$ allele, suggesting that the nine populations do not share the same $W$ chromosome with the main ZW group. Mitochondrial 12S rRNA genotyping shows that the P5 population belongs to the main ZW group, whereas the other six populations belong to the XY group. Thus, the Nosaka mountain range running northeast to southwest can be regarded as another boundary area separating the main ZW group, including the P5 population, from the remaining six $Z Z / Z W$ populations. These remaining six $\mathrm{ZZ/ZW}$ populations constitute a new group, on the basis of the $A R$ expression of allele $\mathrm{W}$ and mitochondrial $12 \mathrm{~S}$ rRNA sequence (Figure 4).

In summary, we identified two distinct geographic boundary areas: the Suzuka-Kii mountain range which separates the western $\mathrm{ZZ} / \mathrm{ZW}$ populations from the eastern $X X / X Y$ populations, and the Nosaka mountain range which separates the main $Z W$ group (including the P5 population) from the other six $\mathrm{ZZ} / \mathrm{ZW}$ populations which constitute a new group that belongs genetically to the XY group (Figure 4). Similar cases in which the distribution area is separated by a mountain range have been shown in other Japanese lower vertebrates. The Suzuka-Kii mountain range separates geographic groups of the freshwater stickleback, Gasterosteus aculeatus, and the Nosaka mountain range is northernmost area of geographic distribution of the Japanese small salamander, Hynobius nebulosus (Watanabe et al., 2003; Matsui et al., 2006). Six populations (P6 and P11-P15) around Lake Biwa belong phylogenetically to the $\mathrm{XY}$ group but are heterogametic in females, as seen previously in the Katata population (Nishioka et al., 1993a, 1994). Further, the $\mathrm{W}$ chromosome allele $b$ of the $A R$ gene is expressed at the same level as that of allele $a$ on the $Z$ chromosome. These are completely different from the main ZW group distributed in northwestern Japan, and the Nosaka mountain range lies between them, providing a geographic boundary. Consequently, the six ZZ/ZW populations should be recognized as a new geographic group, which we have named the 'Neo-ZW group' (Figure 4).

Origin and evolution of the Neo-ZW group

$W$ chromosome: Here, we examine the origins of the $W$ and $\mathrm{Z}$ chromosomes in the Neo-ZW group to determine how the Neo-ZW group differentiated. There are two candidate chromosomes that could provide an origin for the Neo-W chromosome: one is the $W$ chromosome of the main $\mathrm{ZW}$ group, and the other is the $X$ chromosome of the XY group. The $W$ chromosome of the main $Z W$ group is easily rejected as the candidate as it has a dominant female determining function, and is always maternally inherited and accompanied by maternal mitochondria (Nishioka et al., 1993b; Nishioka and Hanada, 1994). If the $W$ chromosome of the Neo group came from the $\mathrm{W}$ chromosome of the main $\mathrm{ZW}$ group, the mitochondrial 12S rRNA haplotype of the Neo-ZW group should be the same as the $\mathrm{ZW}$ group. However, it is in fact the same as the $X Y$ group. In addition, hybrids bearing the $W$ chromosome made between the $X Y$ and main ZW groups are always females (Nishioka and Hanada, 1994). It is unlikely that the $W$ chromosome of the ZW group would be introduced into the $X Y$ group without being accompanied by mitochondria from the ZW group. Conversely, the mitochondrial genotype suggests that the Neo-W came from the $X$ chromosome of the XY group. This is strongly supported by the fact that the $A R$ expression from the Neo-W allele is similar to of the expression from the $X$ chromosome but not similar to that of the main $W$ chromosome. We made gynogenetic diploids from the ZW females of the P13 (Kyoto) population to allow examination of the lethal genes accumulated on the $W$ chromosome of the Neo group by degeneration. The main $W$ chromosome is known to have already accumulated lethal degenerated genes and thus WW embryos die at the tadpole stage after feeding (Ohtani et al., 2003). In contrast, it is evident that the $X$ chromosome has no degenerated lethal genes, as the $X X$ female is normally viable. The results show that one female, no. 1 , had accumulated some lethal degenerated genes on the $W$ chromosome, whereas the other female, no. 2, had not. Therefore, the W chromosomes of the Neo group are still at an early stage of differentiation, and some $\mathrm{W}$ chromosomes may have 
not accumulated degenerated lethal genes as yet. It is therefore plausible that the Neo-W chromosome originated from the $X$ chromosome of the $X Y$ group and is still at an early stage of $\mathrm{W}$ chromosome evolution.

Z chromosome: So, let's now consider the origin of the Neo-Z chromosome. The Neo-Z chromosome has the same haplotypes for two sex-linked genes as seen in the main ZW group and the west Japan group; thus the Neo$Z$ chromosome may have its origin in the homologous no. 7. chromosome from the west Japan group, or the $Z$ chromosome from the main ZW group. However, it could not have originated from the $Y$ chromosome of the $X Y$ group, as lethal genes have already accumulated on the $Y$, as can be seen in the $Y Y$ lethality at an early stage of embryogenesis (H Ohtani, unpublished). Previous isozyme studies on the Katata population (belonging to the Neo-group) located at the west side of Lake Biwa show that some enzyme loci have introgressed from the west Japan group into the Katata population (Nishioka et al., 1993a). Therefore, the Neo-Z chromosome is likely to have derived from the homologous no. 7 of the west Japan group and the ZZ males may be $7 / 7$ or $7 / Y$ (7 indicates the homologous no. 7 chromosome of the west Japan group).

Change of heterogametic sex from male to female: On the basis of the above-mentioned results, it is plausible that the Neo-ZW group diverged from the primary $X Y$ group distributed around Lake Biwa through the introduction of frogs from the west Japan group. How, then, did the heterogametic sex change from male to female in the Neo-ZW group? Speculating what happened in the primary population, we hypothesized introgression of the west Japan group into the $X Y$ group. To confirm this hypothesis, we artificially crossed frogs from the $X Y$ group Hamakita population and the west Japan group Hiroshima population. The sex ratio of the reciprocal offspring skewed toward males: from the $\mathrm{XX}$ female $\times$ West male crosses, $55.4 \%$ of frogs were male after metamorphosis, and $68 \%$ were male at the 1-year point. From the West female $\times$ XY male crosses, $61.4 \%$ of post-metamorphosis frogs were males. It is possible that the introduction of frogs from the west Japan population into the $X X / X Y$ population skewed the sex-ratio toward male, and then a new dominant female determiner was recruited from the $X$ chromosome, following which it quickly extended into the population, finally establishing a female heterogametic system to replace the previous male heterogametic system. Similar cases in which a dominant female determiner arose out of the original $\mathrm{XX} / \mathrm{XY}$ system are known in platyfish and houseflies (Rubini et al., 1972; Kallman, 1984). This story matches well with our previous hypothesis of the mechanism of heterogametic sex change from male to female in the primary population of the main ZW group (Ogata et al., 2003). It is thus possible to speculate that heterogametic sex change, from male $(X X / X Y)$ to female $(Z Z / Z W)$, may have occurred twice and independently during speciation of the frog Rana rugosa (Figure 5). This may have occurred by the following process. The first change in the primary population of the main $\mathrm{ZW}$ group was triggered by hybridization between the west Japan and Kanto groups (Ogata et al., 2003). An XX/XY population was derived from this hybrid population, and may have

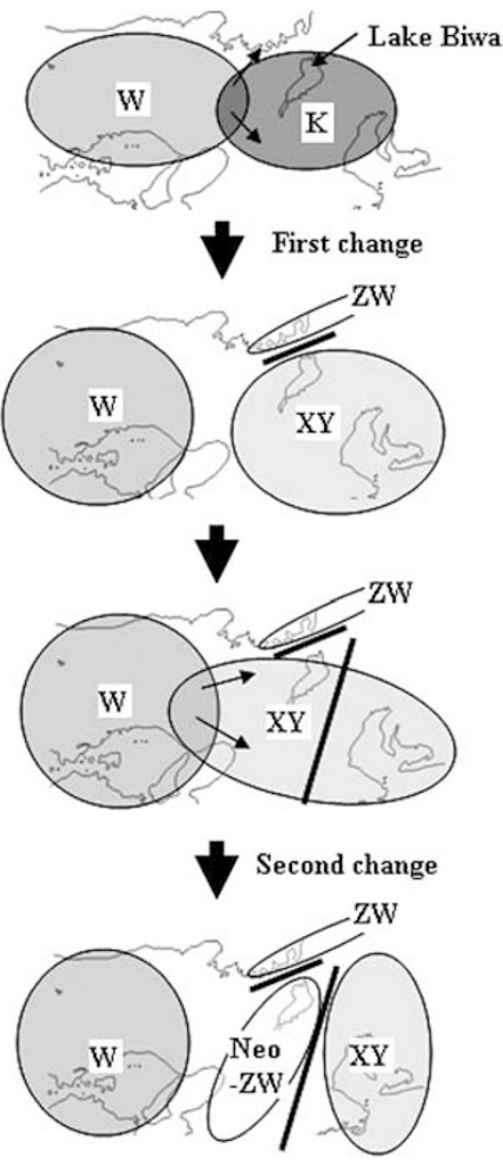

Figure 5 Hypothesized population movements followed by change of heterogametic sex. On the basis of the results of our previous and present studies, we conclude that the heterogametic sex changed from male to female twice and independently in Rana rugosa. Initial hybridization between the west Japan (W) and Kanto (K) populations, both of which have heterogametic males, produced two new groups: in one (ZW), the heterogametic sex changed to female, and in the other $(X Y)$ it remained unchanged. Frogs from the west Japan population(s) then bred with the $\mathrm{XX} / \mathrm{XY}$ populations (XY). Mutual hybridization then changed the heterogametic sex to female, finally creating a new group, Neo-ZW. The two mountain ranges are shown by bold lines: the upper line represents Mt. Nosaka and the lower represents Mt. Suzuka-Kii.

been isolated from the former $\mathrm{ZZ} / \mathrm{ZW}$ population by the Nosaka mountain range. The second change was triggered when west Japan frogs joined the $X X / X Y$ populations, which may have already been isolated from the main $X Y$ group by the Suzuka-Kii mountain range, thus creating the Neo-ZW population. To provide evidence for this evolutionary story regarding the origin of the Neo-ZW group, it is necessary to find more molecular markers that will allow discrimination between the sex chromosomes and elucidate the origins of the Neo-Z and Neo-W chromosomes.

Finally, although it is evident that change of heterogametic sex has occurred often and independently in many vertebrates (Ezaz et al., 2006), no vertebrates have yet been found that have undergone change of heterogametic sex twice or more during their speciation. Therefore, if our hypothesis that change of heterogametic sex occurred twice during speciation of Rana rugosa is true, there must be some special reasons and genetic mechanisms for favoring change of heterogametic sex 
when the inner or outer environment is changed through extending its distribution and meeting other populations. One of the outer reasons acting as a trigger for changing heterogametic sex may be skewing of the sex ratio (Ogata et al., 2003). One of the inner genetic reasons may be the presence of an (as yet unidentified) ovarydetermining gene on sex chromosome 7 . The evidence for this lies in the fact that chromosome 7 was involved in the two previous and independent changes of heterogametic sex.

\section{Acknowledgements}

We thank S Itokuji (Heian Shrine), and M Matsui and K Nishikawa (Kyoto University) for supplying information about the frog habitat around Kyoto.

\section{References}

Bull JJ (1983). Evolution of Sex Determining Mechanisms. The Benjamin/Cummings Publ. Comp. Inc.: Menlo Park, CA, USA.

Ezaz T, Stiglec R, Veyrunes F, Graves JAM (2006). Relationships between vertebrate $\mathrm{ZW}$ and $\mathrm{XY}$ chromosome systems. Curr Biol 16: 736-743.

Hillis DM, Green DM (1990). Evolutionary changes of heterogametic sex in the phylogenetic history of amphibians. J Evol Biol 3: 49-64.

Kallman KD (1984). A new look at sex determination in Poeciliid fishes. In: Turner BJ (eds). Evolutionary Genetics of Fishes. Plenum Publishing: NY, USA. pp 95-165.

Matsui M, Nishikawa K, Utsunomiya T, Tanabe S (2006). Geographic allozyme variation in the Japanese clouded salamander, Hynobius nebulosus (Amphibia: Urodela). Biol J Linn Soc 89: 311-330.

Miura I, Ohtani H, Hanada H, Ichikawa Y, Kashiwagi A, Nakamura M (1997). Evidence for two successive pericentric inversions in sex lampbrush chromosomes of Rana rugosa (Anura: Ranidae). Chromosoma 106: 178-182.

Miura I, Ohtani H, Kashiwagi A, Hanada H, Nakamura M (1996). Structural differences between XX and ZW sex lampbrush chromosomes in Rana rugosa females (Anura: Ranidae). Chromosoma 105: 237-241.

Miura I, Ohtani H, Nakamura M, Ichikawa Y, Saitoh K (1998). The origin and differentiation of the heteromorphic sex chromosomes Z, W, X and Y of the frog Rana rugosa, inferred from the sequences of a sex-linked gene, ADP/ATP translocase. Mol Biol Evol 15: 1612-1619.

Nishioka M, Hanada H (1994). Sex of reciprocal hybrids between the Hamakita ( $X X-X Y$ type) population and the Murakami (ZW-ZZ type) population of Rana rugosa. Sci Rep Lab Amphibian Biol (Hiroshima University) 13: 35-50.

Nishioka M, Hanada H, Miura I, Ryuzaki M (1994). Four kinds of sex chromosomes in Rana rugosa. Sci Rep Lab Amphibian Biol (Hiroshima University) 13: 1-34.

Nishioka M, Kodama Y, Sumida M, Ryuzaki M (1993a). Systematic evolution of 40 populations of Rana rugosa distributed in Japan elucidated by electrophoresis. Sci Rep Lab Amphibian Biol (Hiroshima University) 12: 83-131.

Nishioka M, Miura I, Saitou K (1993b). Sex chromosomes of Rana rugosa with special reference to local differences in sex determining mechanism. Sci Rep Lab Amphibian Biol (Hiroshima University) 12: 55-81.

Ogata M, Lee JY, Kim S, Ohtani H, Sekiya K, Igarashi T et al. (2002). The prototype of sex chromosomes found in Korean population of Rana rugosa. Cytogenet Genome Res 99: 185-193.

Ogata M, Ohtani H, Igarashi T, Hasegawa $\mathrm{Y}$, Ichikawa $\mathrm{Y}$, Miura I (2003). Change of the heterogametic sex from male to female in the frog. Genetics 164: 613-620.

Ohtani H, Miura I, Ichikawa Y (2003). Role of aromatase and androgen receptor expression in gonadal sex differentiation of ZW/ZZ-type frogs, Rana rugosa. Comp Biochem Physiol C Toxicol Pharmacol 134: 215-225.

Ohtani H, Miura I, Hanada H, Ichikawa Y (2000). Alteration of the sex determining system resulting from structural change of the sex chromosomes in the frog Rana rugosa. J Exp Zool 286: 313-319.

Ohtani H, Miura I, Kondo Y, Uchibori M (1997). Amphidiploidy recovers the viability of hybrids of European and East Asian water frogs. I Exp Zool 279: 113-117.

Rubini PG, Franco MG, Vanossi ES (1972). Polymorphisms for heterochromosomes and sutosomal sex-determinants in Musca domestica L.. Atti del IX Congresso Nazionale Italiano di Entomologia 341-352.

Sakisaka Y, Yahara T, Miura I, Kasuya E (2000). Maternal control of sex ratio in Rana rugosa: evidence from DNA sexing. Mol Ecol 9: 1711-1715.

Watanabe K, Mori S, Nishida M (2003). Genetic relationships and origin of two geographic groups pf the freshwater three spine stickleback, 'Hariyo'. Zool Sci 20: 265-274. 